PROCEEDINGS OF THE

AMERICAN MATHEMATICAL SOCIETY

Volume 126, Number 1, January 1998, Pages 67-74

S 0002-9939(98)04487-6

\title{
ON CONFORMAL DISTORTION AND SULLIVAN'S SECTOR THEOREM
}

\author{
EDSON DE FARIA
}

(Communicated by Linda Keen)

\begin{abstract}
Using general bounds on the conformal distortion of univalent maps, we prove a strong version of Sullivan's sector theorem, which gives certain sufficient conditions for an arbitrarily long composition of univalent Herglotz functions to map the upper half-plane into a proper sub-sector.
\end{abstract}

\section{INTRODUCTION}

The purpose of this article is to generalize the sector theorem of D. Sullivan. A simplified statement of this theorem says that a long composition of square-root-like mappings in the upper half-plane $\mathbb{C}^{+}$will map $\mathbb{C}^{+}$into a smaller sector of angle $<\pi$ independent of the length of the composition, provided their poles diverge towards $\pm \infty$ along the real axis at an exponential rate. The sector theorem was used by Sullivan in [8] to establish complex a-priori bounds for renormalization of quadraticlike mappings ( $c f$. also [7]). The robust estimates given by this powerful tool can be used to prove strong compactness properties of certain composition operators acting on analytic mappings, such as the renormalization operators appearing in [8]. The more general version presented here was used by the author in [3] to prove similar results for critical homeomorphisms of the circle.

We proceed to a precise statement of the generalization we have in mind. Given $a, b \in \mathbb{R}$ with $a<b$, let $\mathcal{H}_{a, b}$ be the class of all univalent mappings $\phi$ defined on $\mathbb{C}\left(I_{\phi}\right)=(\mathbb{C} \backslash \mathbb{R}) \cup I_{\phi}$, where $I_{\phi} \supseteq(a, b)$ is some open interval, which preserve both half-planes $\mathbb{C}^{+}, \mathbb{C}^{-}$and are such that $\phi((a, b))=(a, b)$. Members of this class are normalized, univalent Herglotz functions (cf. [4], [9]). We refer to $I_{\phi}$ as the base of $\phi \in \mathcal{H}_{a, b}$ : it is the largest interval containing $(a, b)$ restricted to which $\phi$ is a homeomorphism into the reals. An element $A \in \mathcal{H}_{a, b}$ is a left $\alpha$-root $(0<\alpha<1)$ if there exists $a_{0} \leq a$ such that $A(z)=u\left(z-a_{0}\right)^{\alpha}+v$, where $u, v \in \mathbb{R}$ and the branch of $z \mapsto\left(z-a_{0}\right)^{\alpha}$ is uniquely determined by the requirements $A(a)=a, A(b)=b$. The point $a_{0} \in \mathbb{R}$ is the pole of $A$. Right roots can be similarly defined, but will not be used here. Given a bounded interval $J \subseteq \mathbb{R}$ and any $\lambda>0$, we denote by $J^{\lambda}$ the closed interval centered at the midpoint of $J$ whose length is $(1+\lambda)$-times the length of $J$.

Received by the editors November 21, 1994.

1991 Mathematics Subject Classification. Primary 30C55, 30D05; Secondary 58F23.

Key words and phrases. Non-linearity, conformal distortion, univalent mappings.

This work has been supported by FAPESP Grant 95/3187-4.

(C) 1998 American Mathematical Society 
Theorem 1 (Generalized sector theorem). Let there be given $A_{i}, B_{i} \in \mathcal{H}_{a, b}$, for $i=1,2, \cdots, m$, and constants $\lambda, K, s>0$ and $0<\alpha<1$ satisfying the following conditions:

(a) Each $A_{i}$ is a left $\alpha_{i}$-root with $\alpha_{i} \leq \alpha$ and pole at $a_{i}$, where $a_{1}=a$ and $a_{i}<a$ for all $i \geq 2$.

(b) There exists a finite sequence $1=i_{0}<i_{1}<\cdots<i_{q}=m$ with $i_{n+1}-i_{n} \leq s$ such that, if $d_{n}=\min \left\{\left|a_{i}-a\right|: i_{n} \leq i<i_{n+1}\right\}$, then $\sum_{j \geq n} d_{j}^{-1} \leq K d_{n}^{-1}$ for all $n$.

(c) For all $i \geq 2$, if $I_{i}$ is the base of $B_{i}$ then $B_{i}\left(I_{i}\right) \supseteq\left[a_{i}, b\right]^{\lambda}$, and, setting $J_{i}=B_{i}^{-1}\left(\left[a_{i}, b\right]\right)$, one has $J_{i}^{\lambda} \subseteq I_{i}$ also.

Under these assumptions, there exists a positive angle $\theta=\theta(\alpha, s, K, \lambda)$ such that the image of the upper half-plane by the composition $A_{m} B_{m} \cdots A_{i} B_{i} \cdots A_{1} B_{1}$ is contained in the sector $0 \leq \arg (z-a) \leq \pi-\theta$.

A comparison with Sullivan's original statement in [8] reveals that the main difference lies in hypothesis (b). In Sullivan's version, it is required that the poles $a_{i}$, following their order of occurrence in the composition, be grouped together into scales, each containing a bounded number of poles, and that the scales diverge monotonically towards $-\infty$ at an exponential rate. By contrast, our hypothesis (b) is somewhat more flexible, since it allows for some mixing of such scales. We also allow roots with arbitrary angles $(\leq \pi \alpha<\pi)$, not just square-roots as in the original version.

While the essential ideas of the proof of Theorem 1 remain the same as in the original theorem (Koebe distortion, Schwarz's Lemma, trapping the orbit of a point by means of Poincaré neighborhoods), the actual details of the argument are much more involved. All further tools required in the proof are developed in $\S 2$. The complete proof of Theorem 1 is then given in $\S 3$.

\section{NON-Linearity AND ANGLE Distortion}

Before proving Theorem 1, we recall some classical geometric notions about conformal mappings, and derive some consequences from them in the form of a few lemmas. Let $\phi: \Omega \rightarrow \mathbb{C}$ be a univalent mapping of a domain $\Omega \subseteq \mathbb{C}$, and let $D \subseteq \Omega$ be closed and convex. We define the total non-linearity of $\phi$ on $D$ to be the quantity

$$
N_{\phi}(D)=\operatorname{diam}(D) \sup _{z \in D}\left|\frac{\phi^{\prime \prime}(z)}{\phi^{\prime}(z)}\right|
$$

The notation is taken from [6]. The total non-linearity is a monotone non-decreasing function of $D$, and vanishes identically when $\phi$ is linear. The reason for assuming $D$ convex in the definition is the following. Since for $z_{1}, z_{2} \in D$ the line segment $\left[z_{1}, z_{2}\right]$ is contained in $D$,

$$
\log \left|\frac{\phi^{\prime}\left(z_{1}\right)}{\phi^{\prime}\left(z_{2}\right)}\right| \leq \int_{\left[z_{1}, z_{2}\right]}\left|\frac{\phi^{\prime \prime}(z)}{\phi^{\prime}(z)}\right||d z| \leq N_{\phi}(D)
$$

Therefore,

$$
\sup _{z_{1}, z_{2} \in D}\left|\frac{\phi^{\prime}\left(z_{1}\right)}{\phi^{\prime}\left(z_{2}\right)}\right| \leq \exp \left\{N_{\phi}(D)\right\}
$$


In other words, the total non-linearity bounds ratios of derivatives on convex domains.

When bounding the non-linearity, the main tool at hand is Koebe's distortion lemma, which states that $\left|\phi^{\prime \prime}(z) / \phi^{\prime}(z)\right| \leq 4 / \operatorname{dist}(z, \partial \Omega)$. We shall need also Koebe's one-quarter theorem: If $D \subseteq \Omega$ is a disk with center $z$, then $\phi(D)$ contains a disk about $\phi(z)$ of diameter $\frac{1}{4}\left|\phi^{\prime}(z)\right| \operatorname{diam}(D)$. Both results are corollaries of the distortion theorem of Koebe (cf. [1], [2]). Yet another tool we will use is Schwarz's lemma in the invariant formulation due to Pick: Any holomorphic self-mapping of a hyperbolic Riemann surface $X$ weakly contracts the hyperbolic (or Poincaré) metric of $X$.

Our first lemma gives a simple estimate on how an arbitrary element of $\mathcal{H}_{a, b}$ distorts angles measured from $a$. Here and throughout we write $\theta(z)=\pi-\arg (z-a)$ to denote the angle that $z-a$ forms with the semi-axis $\{x \leq a\}$. Note that $|\operatorname{Im} z|=$ $|z-a||\sin \theta(z)|$.

Lemma 2. If $\phi \in \mathcal{H}_{a, b}$ and if $D$ is any closed convex set with $a \in D \subseteq \mathbb{C}\left(I_{\phi}\right)$, then for all $z \in D$ we have $|\sin \theta(\phi(z))| \geq \exp \left\{-N_{\phi}(D)\right\}|\sin \theta(z)|$.

Proof. As $\phi$ maps $\mathbb{C}^{+}$into itself, the Schwarz-Pick lemma yields

$$
\operatorname{Im} \phi(z) \geq\left|\phi^{\prime}(z)\right| \operatorname{Im} z
$$

for all $z \in \mathbb{C}^{+}$(consider the upper half-plane with its Poincaré metric). Therefore, for all $z \in D \cap \mathbb{C}^{+}$, we have by (2) and the mean value inequality

$$
\sin \theta(\phi(z))=\frac{\operatorname{Im} \phi(z)}{|\phi(z)-a|} \geq \frac{\left|\phi^{\prime}(z)\right||z-a|}{|\phi(z)-a|} \sin \theta(z) \geq \exp \left\{-N_{\phi}(D)\right\} \sin \theta(z),
$$

and the proof is obviously the same for $z \in D \cap \mathbb{C}^{-}$.

In $\S 3$ we use the following property of Herglotz mappings, which we call Poincaré neighborhood trapping. Consider $\mathbb{C}((a, b))$ with its Poincaré metric $\rho$, and let $\gamma$ denote the segment $(a, b)$, a geodesic in this metric. By the Schwarz-Pick lemma, each element of $\mathcal{H}_{a, b}$ maps each Poincaré neighborhood $\mathcal{P}(r)=\{z \in \mathbb{C}((a, b))$ : $\rho(z, \gamma) \leq r\}$ into itself.

Note that $\partial \mathcal{P}(r)$ consists of a circular arc with endpoints $a, b$ in the upper halfplane union its mirror image in the lower half-plane (to see why, map $\mathbb{C}((a, b))$ conformally onto $\mathbb{C} \backslash \mathbb{R}^{+}$by a Möbius transformation sending $(a, b)$ onto the positive real axis, and then map $\mathbb{C} \backslash \mathbb{R}^{+}$conformally onto the upper half-plane using the map $z \mapsto i \sqrt{z})$. In particular, each $z \in \partial \mathcal{P}(r)$ views $\gamma$ under the same angle $\omega=\omega(r)$ (see Figure 1).

Lemma 3. Let $\mathcal{P}_{z}$ be the smallest of the Poincaré neighborhoods $\mathcal{P}(r)$ containing a given point $z$ in the upper half-plane, and let $\mathcal{P}_{z}^{+}=\mathcal{P}_{z} \cap \mathbb{C}^{+}$. Then we have $\operatorname{diam} \mathcal{P}_{z}^{+}=|z-b| / \sin \theta(z)$.

Proof. Apply the law of sines to the triangle $a z b$.

Finally, we will need the following specific geometric property of left roots.

Lemma 4. There exists a continuous function $\Theta:(0,1) \times(0,1) \rightarrow \mathbb{R}^{+}$with the following property. Let $A \in \mathcal{H}_{a, b}$ be a left $\alpha$-root with pole $a_{0}<a$, let $0<R<$ $\left|a_{0}-a\right|$ and let $W$ be the Euclidean convex-hull of $\{a\} \cup\left\{z \in \mathbb{C}^{+}:\left|z-a_{0}\right| \leq R\right\}$. Then $A(W)$ contains the triangle in the upper half-plane with base $\left[A\left(a_{0}\right), a\right] \subseteq \mathbb{R}$ and angles $\pi \alpha$ at $A\left(a_{0}\right)$ and $\Theta\left(\alpha, R /\left|a_{0}-a\right|\right)$ at a, respectively. 


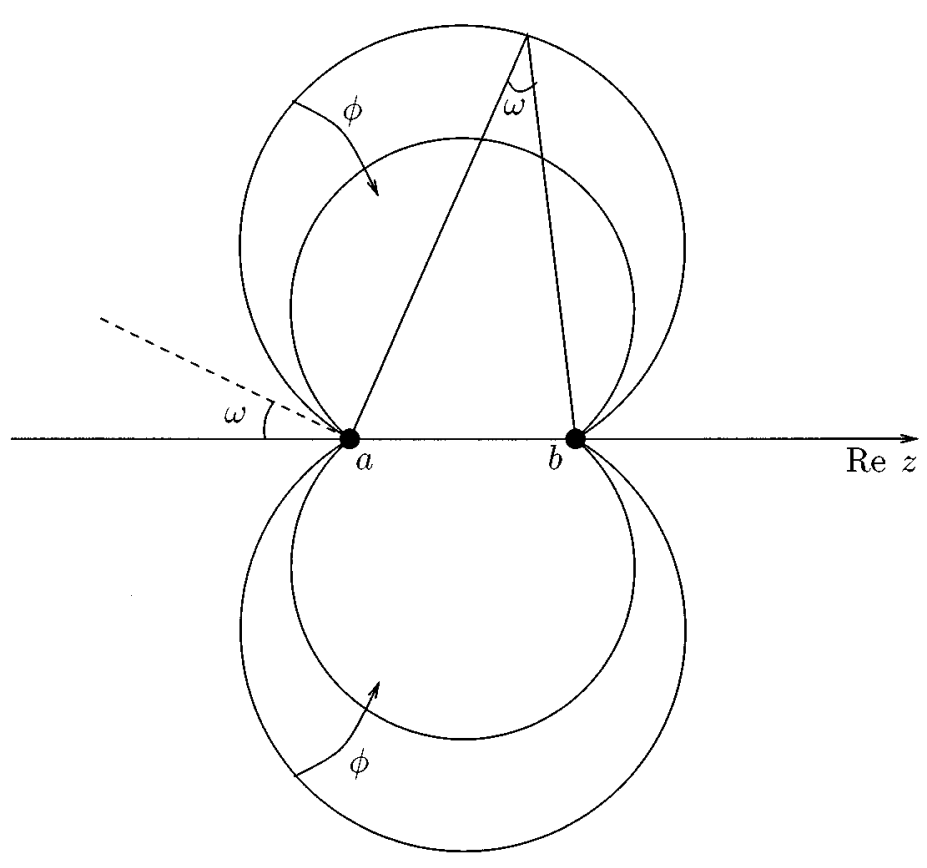

Figure 1

Proof. Recall that $A(z)=u\left(z-a_{0}\right)^{\alpha}+v$, where $u, v$ are determined by the normalization conditions $A(a)=a, A(b)=b$. We first remark that $\theta(A z)>\theta(z)$ for all $z \in \mathbb{C}^{+}$. To see this, let $L_{\varphi}=\left\{a+t e^{i \varphi}: t>0\right\} \subseteq \mathbb{C}^{+}$be a half-line through $a$, and let $H_{\varphi}$ be the component of $\mathbb{C}^{+} \backslash L_{\varphi}$ which lies to the right of $L_{\varphi}$. Computing the curvature of $A\left(L_{\varphi}\right)$, we see that $A\left(L_{\varphi}\right)$ is a convex curve tangent to $L_{\varphi}$ at $a$ and $A\left(L_{\varphi}\right) \subseteq H_{\varphi}$. Therefore $\theta(A z)>\varphi=\theta(z)$ for all $z \in L_{\varphi}$.

Now, to prove the lemma, it suffices to bound $\theta(A z)$ from below for all $z \in$ $\partial W \cap \mathbb{C}^{+}$. Let $\omega=\arcsin \left\{R /\left|a-a_{0}\right|\right\}$ and note that $\partial W \cap \mathbb{C}^{+}=\gamma^{\prime} \cup \gamma^{\prime \prime}$, where $\gamma^{\prime}$ is the circular arc centered at $a_{0}$ with endpoints $x=a_{0}-R$ and $y=a_{0}+R e^{i\left(\frac{\pi}{2}-\omega\right)}$ and $\gamma^{\prime \prime}$ is the line segment joining $y$ to $a$. Then by the above remark we have $\theta(A z)>\omega$ for all $z \in \gamma^{\prime \prime}$. Since $A\left(\gamma^{\prime}\right)$ is a circular arc centered at $A\left(a_{0}\right)$, to bound $\theta(A z)$ from below for $z \in \gamma^{\prime}$ it suffices to do so at the endpoints of $\gamma^{\prime}$, and in fact only at $x$ because $y \in \gamma^{\prime \prime}$ already. Using the equality $v-a=-u\left(a-a_{0}\right)^{\alpha}$, we get

$$
\tan \theta(A x)=\frac{\left|u R^{\alpha} \sin \pi \alpha\right|}{\left|u R^{\alpha} \cos \pi \alpha-u\left(a-a_{0}\right)^{\alpha}\right|}=\frac{\sin \pi \alpha}{\left(\frac{a-a_{0}}{R}\right)^{\alpha}-\cos \pi \alpha} .
$$

Hence, we can define $\Theta(\cdot, \cdot)$ by the formula

$$
\Theta(\alpha, \beta)=\min \left\{\arcsin \beta, \arctan \left(\frac{\sin \pi \alpha}{\beta^{-\alpha}-\cos \pi \alpha}\right)\right\}
$$




\section{Proof of the Generalized SECTOR THEOREM}

In this section we prove Theorem 1 . In the proof $C_{0}, C_{1}, \cdots$ are positive constants depending only on the parameters $\alpha, s, K, \lambda$ of the statement. Regarding hypothesis (b), let $j_{n}$ be the smallest $i$ in the interval $i_{n} \leq i<i_{n+1}$ such that $\left|a_{i}-a\right|=d_{n}$.

Start with any point $z_{1} \in \mathbb{C}^{+}$, and set $z_{i+1}=A_{i} B_{i}\left(z_{i}\right)$ for $i=1,2, \cdots, m$. Our goal is to show that $\theta\left(z_{m+1}\right) \geq \theta$, with $\theta$ as stated. Since we always have $\theta\left(z_{2}\right) \geq(1-\alpha) \pi$, we may assume $m>1$; otherwise there is nothing to prove. Let $U_{i}, i \geq 2$, be a square sitting in the upper half-plane, one of its sides being the interval $J_{i}^{\lambda / 2} \subseteq J_{i}^{\lambda}$. We have $\operatorname{diam} U_{i}=\sqrt{2}\left(1+\frac{\lambda}{2}\right)\left|J_{i}\right|$, while the distance between $U_{i}$ and $\partial I_{i}$ is $\geq \frac{\lambda}{4}\left|J_{i}\right|$. It follows from (1) and Koebe's distortion lemma that $N_{B_{i}}\left(U_{i}\right) \leq C_{0}$, where $C_{0}$ is a constant depending only on $\lambda$. Let $V_{i}$ be the rectangle $U_{i} \backslash\{z: \operatorname{Re} z>a\}$, and observe that $\theta(z) \leq \pi / 2$ for all $z \in V_{i}$.

Lemma 5. There exists $\theta_{1}>0$, depending only on $\alpha$ and $\lambda$, such that $A_{i} B_{i}\left(V_{i}\right)$ contains the triangle $T_{i}$ in the upper half-plane with base $\left[A_{i} a_{i}, a\right] \subseteq \mathbb{R}$ and angles $\pi \alpha_{i}$ and $\theta_{1}$ respectively at $A_{i} a_{i}$ and $a$.

Proof. Since there exists $a<\xi_{i}<b$ such that $B_{i}^{\prime}\left(\xi_{i}\right)=1$ and the non-linearity of $B_{i}$ over $U_{i}$ is bounded by $C_{0}$, we see from (2) that $C_{1}^{-1} \leq\left|B_{i}^{\prime}(x)\right| \leq C_{1}$ for all $x \in J_{i}$, where $C_{1}=e^{C_{0}}$. From hypothesis (c), we find $0<C_{2}<1$ depending only on $\lambda$ such that the disk $D_{i}$ of radius $C_{2}\left|B_{i}^{-1} a_{i}-a\right|$ about $B_{i}^{-1}\left(a_{i}\right)$ is contained in $V_{i}$. Let $H_{i} \subseteq V_{i}$ be the Euclidean convex hull of $\{a\} \cup D_{i}$, and note that $H_{i}=\bigcup_{x \in\left[B_{i}^{-1} a_{i}, a\right]} D_{x}$, where $D_{x}$ is the disk of center $x$ and radius $r_{x}=C_{2}|x-a|$. By Koebe's one-quarter theorem, $B_{i}\left(D_{x}\right)$ contains the disk $D_{x}^{\prime}$ of center $B_{i}(x) \in\left[a_{i}, a\right]$ and radius

$$
R_{x}=\frac{1}{4} r_{x}\left|B_{i}^{\prime}(x)\right| \geq \frac{C_{2}}{4 C_{1}}|x-a| .
$$

Viewed from $a$, the disk $D_{x}^{\prime}$ comprises an angle $2 \theta_{x}$, where

$$
\sin \theta_{x}=\frac{R_{x}}{\left|B_{i}(x)-a\right|} \geq \frac{C_{2}}{4 C_{1}^{2}} .
$$

Therefore $B_{i}\left(H_{i}\right)$ contains the Euclidean convex hull $H_{i}^{\prime}$ of $\{a\} \cup D_{i}^{\prime}$, where $D_{i}^{\prime}$ is the disk of center $a_{i}$ and radius $\left(C_{2} / 4 C_{1}^{2}\right)\left|a_{i}-a\right|$. The result now follows if we apply Lemma 3 to $A=A_{i}$ and $W=H_{i}^{\prime} \cap \mathbb{C}^{+}$, taking $\theta_{1}=\inf _{0<\alpha^{\prime} \leq \alpha} \Theta\left(\alpha^{\prime}, C_{2} / 4 C_{1}^{2}\right)>$ 0 .

This lemma shows that, if $z \in \mathbb{C}^{+} \backslash V_{i}$, then $\theta\left(A_{i} B_{i}(z)\right) \geq \theta_{1}$, for all $i \geq 2$. In what follows we assume, as we may, that $\theta_{1} \leq \pi / 4$. Let $j$ be the smallest index $\geq 2$ such that $z_{i} \in V_{i}$ for all $i \geq j$ (if no such $j$ exists, then $\theta\left(z_{m+1}\right) \geq \theta_{1}$ and we are done). Take the smallest $j_{n} \geq j$ and note that $t=j_{n}-j \leq 2 s$, from hypothesis (b). Our aim is to control the loss of angle when we apply to $z_{j}$ the next $t$ factors $A_{j} B_{j}, A_{j+1} B_{j+1}, \cdots, A_{j_{n}-1} B_{j_{n}-1}$.

As we know, $z_{j}$ belongs to $V_{j}^{*}=V_{j} \backslash\left\{z: 0 \leq \theta(z)<\theta_{1}\right\}$. Let $Q_{j}$ be the square of Figure 2 containing $V_{j}^{*}$. We have $\operatorname{diam} Q_{j} \leq \sqrt{2} \operatorname{diam} V_{j} \leq C_{3}\left|a_{j}-a\right|$. Moreover, by hypothesis (c), the base of $\phi_{j}=A_{j} B_{j}$ contains intervals of length $\geq C_{4}\left|a_{j}-a\right|$ on both sides of $(a, b)$, whence the distances from its endpoints to $Q_{j}$ are $\geq C_{5}\left|a_{j}-a\right| \sin \theta_{1}$ (this uses the fact that $\sin \theta_{1} \leq \cos \theta_{1}$, coming from $\left.\theta_{1} \leq \pi / 4\right)$. Hence, $N_{\phi_{j}}\left(Q_{j}\right) \leq C_{6}\left(\sin \theta_{1}\right)^{-1}$, again by (1) and Koebe's distortion 


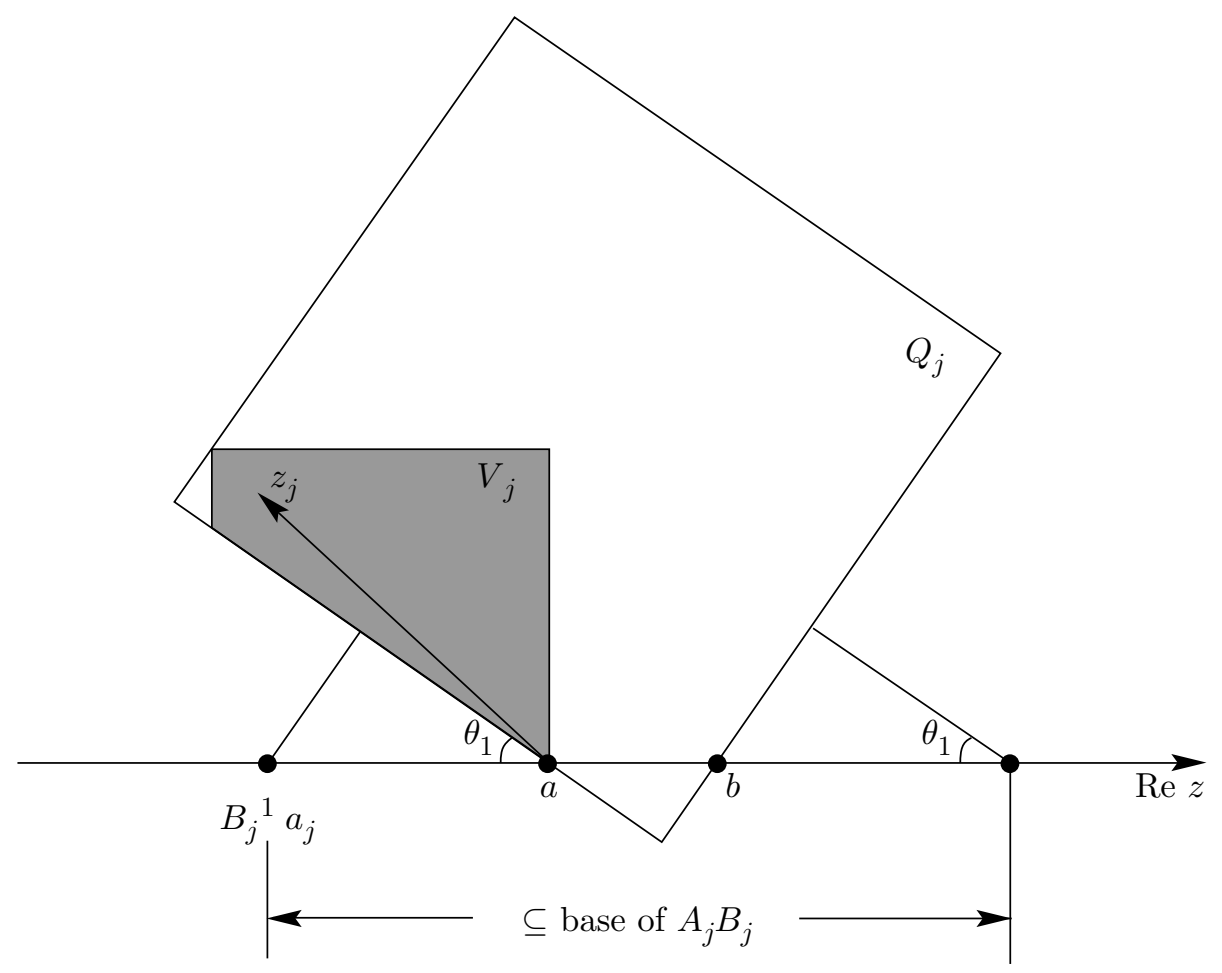

Figure 2

lemma. Therefore, by Lemma 2, we have

$$
\sin \theta\left(z_{j+1}\right) \geq \exp \left\{-C_{6}\left(\sin \theta_{1}\right)^{-1}\right\} \sin \theta\left(z_{j}\right) .
$$

Defining $0<\theta_{k}<\theta_{1}$ recursively by

$$
\sin \theta_{k+1}=\exp \left\{-C_{6}\left(\sin \theta_{k}\right)^{-1}\right\} \sin \theta_{k},
$$

for $k=2, \cdots, t$, and repeating the argument leading to (3) another $t-1$ times, we get $\theta\left(z_{j_{n}}\right) \geq \theta_{t}$, which is a constant still depending only on the parameters in the statement.

Now we appeal to Poincaré-neighborhood trapping in order to control the loss of angle due to the remaining factors. Let $\mathcal{P}$ be the smallest of the Poincaré neighborhoods $\mathcal{P}(r)$ of $(a, b)$ containing $V_{j_{n}} \backslash\left\{z: 0 \leq \theta(z)<\theta_{t}\right\}$, and let $\mathcal{P}^{+}=$ $\mathcal{P} \cap \mathbb{C}^{+}$. From Lemma 3 we know that $2 R=\operatorname{diam} \mathcal{P}^{+}=O\left(d_{n}\right)$. Note that since $\theta_{t} \leq \pi / 4$, we have $R \geq|a-b| / \sqrt{2}$, which is used in the proof of the following lemma.

Lemma 6. For each $i \geq j_{n}$, we have $\operatorname{dist}\left(a_{i}, \mathcal{P}^{+}\right) \geq C_{7}\left|a_{i}-a\right|$.

Proof. Let $h$ be the distance from the center of $\mathcal{P}^{+}$to the real axis, let $\Delta_{i}$ be the distance from $a_{i}$ to the midpoint of $(a, b)$, and let $\delta_{i}=\operatorname{dist}\left(a_{i}, \mathcal{P}^{+}\right)$. Then 
elementary geometry gives us

$$
\frac{\delta_{i}}{\left|a_{i}-a\right|} \geq \frac{\delta_{i}}{\Delta_{i}}=\frac{1-\left(\frac{|a-b|}{2 \Delta_{i}}\right)^{2}}{\frac{R}{\Delta_{i}}+\sqrt{1+\left(\frac{h}{\Delta_{i}}\right)^{2}}}
$$

By hypothesis (b) we have $\left|a_{i}-a\right| \geq K^{-1} d_{n} \geq C_{8} R$ for all $i \geq j_{n}$. Therefore $h<R \leq C_{8}^{-1} \Delta_{i}$, and this bounds the denominator of (4) from above. At the same time,

$$
\Delta_{i}=\left|a_{i}-a\right|+\frac{1}{2}|a-b| \geq\left(1+C_{8} \sqrt{2}\right) \frac{|a-b|}{2},
$$

which in turn bounds the numerator of (4) from below.

For each $i \geq j_{n}$, let $\left(x_{i}^{+}, x_{i}^{-}\right) \subseteq \mathbb{R}$ be the base of $\phi_{i}=A_{i} B_{i}$. By hypothesis (c), we have $\left|x_{i}^{-}-a\right| \geq C_{9}\left|a_{i}-a\right|$ and $\left|x_{i}^{+}-b\right| \geq C_{9}\left|a_{i}-a\right|$. Hence the same method used in the proof of Lemma 6 gives also

$$
\operatorname{dist}\left(x_{i}^{ \pm}, \mathcal{P}^{+}\right) \geq C_{10}\left|a_{i}-a\right| .
$$

Therefore, once again by Koebe's distortion lemma, we see that

$$
N_{\phi_{i}}\left(\mathcal{P}^{+}\right) \leq C_{11} d_{n} /\left|a_{i}-a\right|
$$

Since $\phi_{i}\left(\mathcal{P}^{+}\right) \subseteq \mathcal{P}^{+}$for all $i \geq j_{n}$, applying Lemma 2 recursively together with (6) yields

$$
\begin{aligned}
\sin \theta\left(z_{m+1}\right) & \geq\left(\prod_{i=j_{n}}^{m} \exp \left\{-N_{\phi_{i}}\left(\mathcal{P}^{+}\right)\right\}\right) \sin \theta\left(z_{j_{n}}\right) \\
& \geq \exp \left\{-C_{11} d_{n} \sum_{i=j_{n}}^{m}\left|a_{i}-a\right|^{-1}\right\} \sin \theta\left(z_{j_{n}}\right) .
\end{aligned}
$$

But here, using the full strength of hypothesis (b), we have

$$
\sum_{i=j_{n}}^{m}\left|a_{i}-a\right|^{-1} \leq s \sum_{k \geq n} d_{k}^{-1} \leq K s d_{n}^{-1} .
$$

Taking this back to (7), we get at last $\sin \theta\left(z_{m+1}\right) \geq \exp \left\{-C_{11} K s\right\} \sin \theta_{t}$. This completes the proof of Theorem 1.

\section{ACKNOWLEDGEMENTS}

I wish to thank Welington de Melo for several conversations on these and related matters. I am also grateful to Nils Tongring for reminding me of Carathéodory's beautiful classic [2]. This paper was written in part during visits to the Forschungsinstitut für Mathematik (ETH-Zürich) and the Institut des Hautes Études Scientifiques (Bures-sur-Yvette). I want to thank both institutions for their warm hospitality and generous support. 


\section{REFERENCES}

1. L. Ahlfors, Conformal invariants: topics in geometric function theory, McGraw-Hill, 1973. MR 50:10211

2. C. Carathéodory, Conformal representation, Cambridge University Press, 1952. MR 13:734a

3. E. de Faria, Proof of universality for critical circle mappings, Ph.D. Thesis, CUNY, 1992.

4. H. Epstein, Fixed points of composition operators II, Nonlinearity 2 (1989), 305-310. MR 90j:58086

5. M. Flexor, Théoreme du secteur d'après D. Sullivan, Prepublication UPS Orsay (1992).

6. C. McMullen, Area and Hausdorff dimension of Julia sets of entire functions, Trans. Amer. Math. Soc. 300 (1987), 329-342. MR 88a:30057

7. W. de Melo and S. van Strien, One dimensional dynamics, Springer-Verlag, Berlin and New York, 1993. MR 95a:58035

8. D. Sullivan, Bounds, quadratic differentials and renormalization conjectures, Mathematics into the Twenty-First Century, Amer. Math. Soc. Centennial Publication, vol.2, Amer. Math. Soc., Providence, RI, 1991, pp. 417-466. MR 93k:58194

9. G. Valiron, Fonctions analytiques, Presses Universitaires de France, Paris, 1954. MR 15:861e

Instituto de Matemática e Estatística, Universidade de São Paulo, Rua do Matão 1010, Butantã CEP05508-900 São Paulo SP, Brasil

E-mail address: edson@ime.usp.br 